\title{
New directions for nominalist philosophers of mathematics
}

\author{
Charles Chihara
}

Received: 1 June 2008 / Accepted: 16 June 2008 / Published online: 27 March 2009

(C) The Author(s) 2009. This article is published with open access at Springerlink.com

\begin{abstract}
The present paper will argue that, for too long, many nominalists have concentrated their researches on the question of whether one could make sense of applications of mathematics (especially in science) without presupposing the existence of mathematical objects. This was, no doubt, due to the enormous influence of Quine's "Indispensability Argument", which challenged the nominalist to come up with an explanation of how science could be done without referring to, or quantifying over, mathematical objects. I shall admonish nominalists to enlarge the target of their investigations to include the many uses mathematicians make of concepts such as structures and models to advance pure mathematics. I shall illustrate my reasons for admonishing nominalists to strike out in these new directions by using Hartry Field's nominalistic view of mathematics as a model of a philosophy of mathematics that was developed in just the sort of way I argue one should guard against. I shall support my reasons by providing grounds for rejecting both Field's fictionalism and also his deflationist account of mathematical knowledge_-doctrines that were formed largely in response to the Indispensability Argument. I shall then give a refutation of Mark Balaguer's argument for his thesis that fictionalism is "the best version of anti-realistic anti-platonism".
\end{abstract}

Keywords Nonstandard analysis · Indispensability Argument · Nominalism . Fictionalism · Group theory

C. Chihara $(\varangle)$

Department of Philosophy, University of California,

Berkeley, CA 94720, USA

e-mail: charles1@socrates.berkeley.edu 


\section{Introduction}

Philosophers of mathematics classified as 'nominalists' reject the Platonic idea that the mathematician is exploring, and providing the world with information about, a realm of "nonphysical objects": the numbers, sets, functions and the like that mathematical theorems are supposedly about. ${ }^{1}$ Nominalists believe that attempting to understand the nature of mathematics guided by such a conception is hopeless. Instead, they seek to achieve their goals by studying the way mathematics is used and developed in science and engineering and also by investigating both the history of mathematics and current mathematical practices-without the assumption that such things as mathematical objects truly exist. ${ }^{2}$

The present paper will argue that, for too long, many nominalists have concentrated their researches on the question of whether one could make sense of applications of mathematics (especially in science) without presupposing the existence of mathematical objects. This was, no doubt, due to the enormous influence of Quine's "Indispensability Argument", ${ }^{3}$ which challenged the nominalist to come up with an explanation of how science could be done without referring to, or quantifying over, mathematical objects. But how mathematics is applied in science and engineering is only one aspect of mathematical practice that requires investigating. It is true that I try to characterize the nature of mathematics in a way that is consistent with an overall account of science that is both widely accepted by experts and also consistent with our knowledge of the world. ${ }^{4}$ But, from my perspective, the goal of philosophy of mathematics is to provide an accurate "Big Picture" account of the essential nature of mathematics, as it is actually practiced. ${ }^{5}$ So I see no reason why philosophers should focus all their attention to just applications of mathematics in science.

I shall admonish nominalists to enlarge the target of their investigations to include the many uses mathematicians make of concepts such as structures and models to advance pure mathematics. I shall illustrate my reasons for admonishing nominalists to strike out in these new directions by using Hartry Field's nominalistic view of mathematics as a model of a philosophy of mathematics that was developed in just the sort of way I argue one should guard against. I shall support my reasons by providing grounds

\footnotetext{
1 The mathematician G. H. Hardy expressed a Platonic view of mathematics when he wrote: "I believe that mathematical reality lies outside us, and that our function is to discover or observe it, and that the theorems which we prove, and which we describe grandiloquently as our 'creations' are simply our notes of our observations" (Hardy 1941, pp. 63-64).

2 For a sympathetic account of nominalism as I view it, see (Chihara, 2004, Chap. 6), especially Sect. 2.

3 One finds, in the literature, references to the Quine-Putnam Indispensability Argument because Hilary Putnam has put forward a version of Quine's argument that has been widely discussed (see Chihara 2004, pp. 123-127). Other philosophers have developed versions of Quine's argument (see Chihara 2004, pp. 126-127, for another example).

4 The idea is that philosophy is the search for a coherent account of the world and our place in it. Thus, philosophers of mathematics should seek an understanding of mathematics that is consistent with the other views we accept about the universe and about us. For a clearer and fuller explanation of my view of the nature of philosophy of mathematics, see the introduction to my (Chihara 2004), where a more detailed explanation of the view is given in the context of presenting my view of what philosophy is.

5 What I mean by a "Big Picture" account is explained in detail (Chihara 2004, p. 1).
} 
for rejecting both Field's fictionalism and also his deflationist account of mathematical knowledge - doctrines that were formed largely in response to the Indispensability Argument. I shall then give a refutation of Mark Balaguer's argument for his thesis that fictionalism is "the best version of anti-realistic anti-platonism".

\section{Field's responses to Quine's argument}

One of the most widely discussed arguments for a Platonic view of mathematics is what is called "Quine's indispensability argument". I call the original form of the argument "Quine's challenge to the nominalist" because Quine can be regarded as challenging the nominalist to specify a system of mathematics that would be both adequate for the needs of the scientist and also nominalistic, in so far as it does not require the user to presuppose the existence of mathematical objects. If the challenge could not be met, then Quine could plausibly maintain that we have good reasons for believing that mathematical objects exist. Subsequently, many closely related forms of the "indispensability argument" have been proposed and defended. ${ }^{6}$ Much recent work in the philosophy of mathematics has focused on various forms of this argument and the published responses to them. Field's account of mathematics is one such response.

Carl Hempel's once defended the thesis that mathematics is "empty of factual content"- a thesis that gave rise to the need to explain how a theory that is "empty of factual content" (as Hempel characterizes mathematics) can be so useful—indeed essential-for the empirical sciences. Hempel responded to this need by espousing the view that mathematics functions in science as a kind of "theoretical juice extractor" by aiding scientists to infer the factual implications of their empirical theories-implications that are, in theory, deducible directly from the empirical laws and statements of the theories. Field came up with a more precise and mathematically attractive version of Hempel's theoretical juice extractor view, the core idea of which is a principle, according to which any nominalistic sentence that can be deduced from a nominalistic theory with the aid of mathematics can also be deduced from the nominalistic theory alone. ${ }^{7}$ Using this principle, it is argued (against Quine) that the nominalist can legitimately use Platonic versions of mathematics in science without incurring a commitment to the existence of mathematical objects. The above principle allows the nominalist to use standard versions of set theory to draw nominalistic conclusions from nominalistic versions of science without presupposing the existence of mathematical objects, because the nominalist need not assume (or presuppose) that the theorems of the mathematical theory being used in this way are true. Field can thus adopt a fictionalist attitude toward mathematics: the theorems of mathematics that apparently refer to all sorts of objects that do not exist in the physical world can be regarded as sentences in works of fiction and not as truths (Field 1980, p. 2).

\footnotetext{
6 See (Chihara, 2004, Chap. 5, Sects. 2 and 3), for a detailed account of various versions of the Indispensability Argument, as well as a discussion of Quine's challenge to the nominalist.

7 See (Chihara, 2004, pp. 109-111), for more details regarding Hempel's idea and how Field produced a more rigorous version of Hempel's idea.
} 


\section{Field's account: some details}

Although the above rough sketch of Field's overall response to the indispensability argument should be sufficient for most readers to follow the essentials of the discussion to follow, some additional details of this response will be noted here, if only to facilitate making certain points more clearly and more precisely.

In this section, it should be understood that the languages and theories to be discussed are to be taken to be the languages and theories of first-order logic. ${ }^{8}$ For the most part, Field takes mathematics to be Zermelo-Fraenkel set theory, since it is widely accepted that any mathematical theory needed in the empirical sciences could be formalized in that set theory. Since he wants to allow the chosen mathematical theory to "speak" of things that the scientific theory discusses, he chooses ZermeloFraenkel set theory with urelements (henceforth 'ZFU'). Now as a first approximation to what he wants his principle (henceforth 'Conservation Principle') ${ }^{9}$ to be, consider:

If $\mathrm{N}$ is a nominalistic theory, then $\mathrm{ZFU}+\mathrm{N}$ is a conservative extension of $\mathrm{N}$ (or as I shall sometimes put it: "ZFU is conservative over N") where:

(1) $\mathrm{ZFU}+\mathrm{N}$ is the theory obtained by conjoining the two theories ZFU and N, taking the vocabulary of the new theory to be the union of the vocabularies of the two theories and taking the assertions of the new theory to be the union of the assertions ${ }^{10}$ of the two theories;

and where:

(2) $\mathrm{T}^{*}$ is a conservative extension of $\mathrm{T}$ if, every theorem of $\mathrm{T}^{*}$ that is a sentence of $\mathrm{T}$ is also a theorem of $\mathrm{T}$.

Thus, the first approximation formulated above tells us that any sentence of the nominalistic theory $\mathrm{N}$ that is derivable in the joint theory $\mathrm{ZFU}+\mathrm{N}$ is derivable in $\mathrm{N}$.

One problem with the above formulation of the Conservation Principle is that $\mathrm{N}$ might in some way contradict ZFU or, in effect, attribute all sorts of strange properties to sets when $\mathrm{N}$ is conjoined with ZFU. For example, N might say "Everything satisfies Newton's Laws", thus implying that sets satisfy Newton's Laws. Of course, $\mathrm{N}$ is not really talking about sets when it asserts that everything satisfies Newton's Laws. So Field suggests that the quantifiers of N should be "relativized" so as to be explicitly about nonmathematical objects. This can be done as follows: introduce into the joint language, a monadic predicate $M$ meaning 'is a mathematical object' and relativize the quantifiers of $\mathrm{N}$ to the nonmathematical objects in the following way: ' $(x)(F x \rightarrow G x)$ ' becomes ' $(x)(-M x \rightarrow(F x \rightarrow G x))$ ' and ' $(\exists x) F x$ ' becomes ' $(\exists x)(-M x \& F x)$ '. As for ZFU, it already talks about nonsets, so we need only add another monadic predicate to the joint vocabulary meaning 'is a set' and then add to its axioms a sentence that says "Every set is a mathematical object", i.e. $(x)(S x \rightarrow M x)$.

The above details are generally omitted in discussions of Field's Conservation Principle because it is seen as a rather minor point and because Field decides in his book

\footnotetext{
8 This is not meant to suggest that Field always restricts his Conservation Principle to first-order logic.

9 This is the principle that Field calls "Principle C" in Chap. 1 of Field (1980).

10 The "assertions of a theory" is a set that is closed under the consequence relation.
} 
not to introduce a special notation for the modified versions of $\mathrm{ZFU}+\mathrm{N}$ (he just assumes that $\mathrm{ZFU}+\mathrm{N}$ is written that way from the start (p. 12)). I provide these details because I wish to highlight a feature of Field's account that is frequently overlooked in discussions of his view: as Field formulates ZFU, set theory appears to be a kind of metaphysical theory. The metaphysical nature of its assertions is made explicit by the fact that, among its theorems, there are assertions of the form ' $(\exists x) M x$ ', thus implying that the theory asserts the existence of mathematical objects. ${ }^{11}$ Because Field believes that mathematical objects do not exist, he is convinced that ZFU cannot be a true theory.

It should be noted that treating mathematical theorems as metaphysical in nature is not new with Field. The two most eminent of the Platonist philosophers of mathematics, namely Kurt Gödel and Willard Quine, believed that the assertions of mathematics are metaphysical in nature. Of course, not all philosophers of mathematics adopt such a position. 12

The version of mathematics presented above is of little interest to one concerned with applications of mathematics, since the vocabularies of the two conjoined theories have essentially nothing in common: there is practically no way that the mathematical theory can "interact" with the nominalistic part, so it is hard to see how such a theory can have much use in science. Thus, Field expands the vocabulary of the mathematical part to include the vocabulary of $\mathrm{N}$ so that the nominalistic vocabulary can appear in such axioms as Separation and Replacement. The expanded mathematical system, ZFU*, can then map nominalistic objects to both pure and "mixed" mathematical objects (See Field 1980, pp. 9-10).

The Conservation Principle can now be stated as follows:

$[\mathrm{CP}]$ If $\mathrm{N}$ is a nominalistic theory, then $\mathrm{ZFU}^{*}+\mathrm{N}$ is a conservative extension of $\mathrm{N}$.

What is one supposed to conclude from Field's reasoning about the Indispensability Argument? Field uses the Conservation Principle to justify one of the more controversial of his theses about mathematics: mathematics, according to Field, is not a "body of truths" and "no part of mathematics is true" (p. viii). Field's justification for adopting this remarkable thesis is tied to his attitude toward the Indispensability Argument. In the beginning of the preface to Science Without Numbers, he asks: "[W]hat good argument is there for regarding standard mathematics as a body of truths?" His answer: "The only non-question-begging arguments I have ever heard for the view that mathematics is a body of truths all rest ultimately on the applicability of mathematics to the physical world ..." (Field 1980, p. viii). It is clear that the arguments Field has in mind here are the various versions of the Indispensability Arguments that his conservation strategy is supposed to undermine. ${ }^{13}$ Convinced that his strategy refutes

\footnotetext{
11 The reason I suggest that ZFU is regarded as a sort of metaphysical theory is not because it has existence assertions, but rather because it seems to assert the existence of mathematical objects-something that metaphysicians typically assert, deny, or argue about.

12 See, for example, my own position on this question in Chihara (2004).

13 Cf. "I believe it becomes clear that there is one and only one serious argument for the existence of mathematical entities, and that is the Quinean argument that we need to postulate such entities in order to
} 
those "non-question-begging arguments", Field concludes that "there is no reason to regard any part of mathematics as true", ${ }^{14}$ and this leads Field to adopt the view that the sentences of mathematics are like those in works of fiction.

In concluding this section, I would like to emphasize two features of Field's account of mathematics that contribute considerably to its attractiveness.

\subsection{Field's "no reinterpretation" account of mathematics}

Field's account of mathematics does not require that mathematical sentences be given any special interpretation in order to be applied in science; nor does it require that a special kind of logic be used in such applications. The nominalist can even employ, for example, ZFU—a first-order theory as standardly understood—in applying mathematics to science. Thus, he writes:

The way that has proved most popular among nominalistically inclined philosophers is to try to reinterpret mathematics - reinterpret it so that its terms and quantifiers don't make reference to abstract entities ... My approach is different: I do not propose to reinterpret any part of classical mathematics ... (Field 1980, p. 2).

This feature of Field's account has been thought to lead to the conclusion that the account fits actual mathematical practice perfectly. Since the nominalist can use the standard classical systems of mathematics to draw nominalistic conclusion from nominalistic theories, there does not appear to be any place for Field's account to conflict with any mathematical practice.

\subsection{Field's fictionalism}

Fictionalism has a substantial history. In the 1970s, I sketched a view about mathematics in which set theory was likened to a work of fiction. The view was a form of Platonism in so far as its adherents felt no intuitionist qualms about using the law of excluded middle, saw nothing wrong with the notion of the set of all real numbers or with impredicative specifications of sets, and did not think that mathematics was concerned with reasoning about mental constructions or strings of symbols. But these theorists did not believe in the existence of mathematical objects. They were not "ontological platonists", but rather were "mythological platonists". They could agree with those theorists who hold that the continuum hypothesis is neither true nor false by regarding the hypothesis as being analogous to the statement that 'Hamlet's nose was 4 1/2 inches long' - an assertion most thinkers would regard as not properly evaluated in terms of truth and falsity. When set theory is regarded in this fictionalist way, one

\footnotetext{
Footnote 13 continued

carry out ordinary inferences about the physical world and in order to do science" (p. 5). Also: "The hardest part of showing that the application of mathematics doesn't require that the mathematics that is applied is true is to show that mathematical entities are dispensable in a way that theoretical entities in science are not ..." (p. viii).

14 (Field, 1980, p. viii), italics mine.
} 
could give explanations of certain sorts of facts that Gödel had used to support various Platonic conclusions. 15

Interestingly, Field expresses sympathy for this early form of fictionalism in his book. ${ }^{16}$ And a very similar kind of fictionalism is espoused there by Field. Since he believed that he could refute the only non-question begging reason for thinking that mathematics is a body of truths, he concluded that there is no good reason for thinking that any part of mathematics is true, commenting:

This is not of course to say that there is something wrong with mathematics; it's simply to say that mathematics isn't the sort of thing that can be appropriately evaluated in terms of truth and falsehood. (Field 1980, p. viii)

\section{The claimed superiority of fictionalism}

The fictionalist view described above has been thought to be superior to all other nominalistic views that have been developed. Specifically, Mark Balaguer writes that "fictionalism is the best version of anti-realistic anti-platonism" (Balaguer 1998, p. 102). The grounds for this remarkable assessment of the relative superiority of fictionalism over all other anti-realistic anti-platonist theories is said to be based upon the fact that all competing nominalistic accounts of mathematics differ from fictionalism not in any ontological way, but "only in the interpretations that they provide for mathematical theory and practice" (p. 102). But then, it is argued, fictionalism can be seen to be superior to its competitors because of two admirable features claimed by Balaguer to be possessed by fictionalism. First, it is asserted that "there is nothing in mathematical practice that runs counter to fictionalism" (Balaguer 1998, p. 103). The idea is that the fictionalist account of mathematics fits perfectly the actual practice of mathematics. This first assertion supports the second: "fictionalism interprets our mathematical theories in a very standard, straightforward, face-value way, [whereas] other versions of anti-realism ... advocate controversial, non-standard, non-face-value interpretations that seem to fly in the face of actual mathematical practice" (Balaguer 1998, p. 102).

\section{Field's deflationist view of mathematical knowledge}

Field's doctrine that the sentences of mathematics are like those in works of fiction is an important feature of his overall view of mathematics. It is this doctrine that motivates his "deflationist" position about mathematical knowledge—a position that attempts to account for "mathematical knowledge" without requiring the possessor of mathematical knowledge to know that any specific mathematical theorem is true. Since these theorems are held to be neither true nor false, it is thought that they could not be known to be true. Thus, in his article "Is Mathematical Knowledge Just Logical Knowledge?", Field claims that "what separates someone who knows lots of math-

\footnotetext{
15 See (Chihara, 1973, Chap. 2, Sect. 2). Leslie Tharp developed and extended some of the ideas of mythological platonism in an attempt to arrive at a general account of the nature of mathematics in Tharp (1989). See, in this connection, Chihara (1989).

16 See (Field, 1980, fn. 4).
} 
ematics from one who knows only a little mathematics is not that the former knows many and the latter knows few of such claims as those that mathematicians commonly provide proofs of ..." (p. 81). What separates them, according to Field, is "empirical knowledge (e.g., about what other mathematicians accept and what they use as axioms)" and, more importantly, knowledge of a purely logical sort (p. 82). Thus, it is claimed that what chiefly distinguishes someone with lots of mathematical knowledge from someone with little is that the former, but not the latter, has lots of knowledge of truths of the form:

(i) It is logically necessary that if $A$, then $B$

and

(ii) It is logically possible that $A .^{17}$

But what does it mean to say of a proposition that 'it is logically necessary or logically possible'? Field tells us that "the modal knowledge which deflationism allows is knowledge of purely logical possibility - deflationism does not allow knowledge of mathematical possibility in an interesting sense" (1989, p. 85, n. 7). As Field uses the modal operator,

It is logically possible that ' $(\exists x)(x$ is a bachelor $\& x$ is married $)$ '

is true. In Field's modal logic, there are no "meaning postulates" that specify "logical" relations among the predicates. Thus, it is logically possible that there are married bachelors (in Field's sense of the operator). In another work, he emphasizes the restricted sense he gives to his modal operator by limiting the logical truths to sentences "true by logical form alone" (Field 1992, pp. 114-115), noting that the logical truths he has in mind are "purely logical".

Let us, then, distinguish two quite different theses being promulgated by Field. There is first the thesis about mathematical truth:

[NT] No part of mathematics is true.

Then there is the deflationist thesis about mathematical knowledge that is in essence:

[MK] What the mathematician knows, that the non-mathematician does not, are modal facts of the form (i) and (ii).

Of course, the two views are related. It is hard to conceive of a philosopher espousing $[\mathrm{MK}]$ if she did not already believe [NT]. A philosopher who accepted [NT] could not believe that the proofs mathematicians produce, in the course of doing what are called "proving a theorem", are proofs of the truth of the theorem proved. Thus, [NT] pushes one to espouse a view according to which the theorems of set theory are not true but only logical consequences of the axioms of the set theory being assumed; and the knowledge obtained as a result of the proof is not knowledge of the truth of the theorem proved but only knowledge of something like:

It is logically necessary that (if A, then T)

17 (Field, 1984, p. 85). 
where $\mathrm{A}$ is some finite set of axioms and $\mathrm{T}$ is the theorem proved. Consequently, $[\mathrm{NT}]$ pushes one toward some such proposition as $[\mathrm{MK}]$ and also provides $[\mathrm{MK}]$ with what plausibility it has. Since Field came to his belief in [NT] largely as a result of his juice extractor response to the Indispensability Argument, it can be seen that the deflationist view of mathematical knowledge was itself formed largely in response to the Indispensability Argument.

\section{Doubts to be raised}

There have been many criticisms of Field's view of mathematics - too many to discuss here. ${ }^{18}$ Still, some nominalists, who are primarily concerned with responding to the various indispensability arguments, may find Field's view of mathematics both promising and plausible. What I shall argue is that this view of mathematics is not so attractive when one is targeting for investigation the many uses of structures in pure mathematics, where reference to, and reasoning in terms of, structures have been found to be strikingly fruitful, even though the value of such uses cannot be adequately understood or explained in terms of the Conservation Principle. ${ }^{19}$ Furthermore, I shall give grounds for rejecting both his fictionalism and his deflationist account of mathematical knowledge, as well as Balaguer's argument for the superiority of fictionalism. However, to explore in a preliminary way reasons for questioning the cogency of Field's views on mathematics, consider the following developments in the history of mathematics.

\section{A new approach to algebra}

Two historians of mathematics have noted the following developments:

[U]ntil the 19th century, algebra was largely the science of (determinate and indeterminate) equations, ${ }^{20}$ whereas in the 19 th century there appeared in it completely new concepts and objects, such as groups, rings, fields, ideals, ... [which] brought about a changed view of the subject matter of algebra. Specifically, the task of algebra was now seen to be the study of systems of arbitrary nature for which there are defined operations with properties more or less similar to those of addition and multiplication of numbers. ${ }^{21}$

In 1930, Bartel van der Vaerden published an influential textbook entitled "Modern Algebra", which articulated the developing view of algebra that was coming into

18 See (Chihara, 2004, Chap. 11).

19 This is not to say that the fictionalist cannot give some sort of explanation of its value in terms of conservatism. I only claim here that such an explanation would not be adequate.

20 It is noted that:

A system of polynomial equations is said to be indeterminate if it has fewer equations than variables, and to be determinate if the number of equations equals or exceeds the number of variables ... The term 'indeterminate' is most commonly applied to systems with fewer equations than variables for which integer or rational solutions are sought. Such systems, also called 'Diophantine', have been particularly influential in the development of algebra ... (Bashmakova and Smirnova 2000, pp. xv-xvi).

21 (Bashmakova and Smirnova, 2000, p. xiii). 
vogue. The historian of mathematics Leo Corry describes this new view of algebra as the structural approach to algebra:

The essence of the structural approach to algebra lies in the recognition that it is mathematically enlightening to conceive a handful of concepts (groups, rings, fields, etc.) as individual "varieties" of the same mathematical "species". ., namely, the species of algebraic structures. With the adoption of this approach, the study of algebraic structures gave algebraic research a new focus, subsuming under it the traditional tasks of the discipline, namely, the study of polynomial forms and polynomial equations, and the problem of the solvability of polynomial equations. Moreover, under the new approach to algebra, this discipline came to cover under its unified scope, the study of other related, but theretofore separated domains of research, particularly algebraic number theory. ${ }^{22}$

The developments being described above had far-reaching and long-lasting consequences in the field. ${ }^{23}$ The subsequent widespread adoption of the structural approach both in algebraic research and in the teaching of the subject yielded many benefits: many open problems were solved in an economic and elegant way, previously solved problems were presented in a new and interesting light, and new mathematically intriguing problems were formulated (Corry 1996, p. 9). In a word, the new approach was mathematically fruitful.

Without attempting any sort of deep analysis of these developments in the subject, one can see one obvious reason why the structural approach has appeared attractive to algebraists: the efficiency with which the subject matter of algebra can be studied and developed in terms of algebraic structures is a real advantage. It is the sort of efficiency that aids mathematicians in learning and teaching the material. This efficiency can be observed in the following way of presenting the fundamentals of algebra.

Imagine a graduate course in abstract algebra that begins with the theory of groups. Such a start would be fitting since the concept of group is both simply expressed and easily grasped, even though it is "one of the most important concepts of modern

22 (Corry, 1996, p. 9). One should not infer from the above that van der Vaerden was solely or even principally responsible for advancing the structural approach to algebra. His textbook "assembled the important results that had been obtained during the last decades of research in its domain of concern and exposed them in a systematic, and didactically clear, fashion" (Corry 1996, p. 8). It should also be noted that the quotation from Bashmakova and Smirnova (2000) emphasizes that, until the 19 th century, algebra was largely the science of both determinate and indeterminate equations, whereas the quotation from Corry (1996) gives no indication of the importance of indeterminate equations for the early algebraists. In the Introduction to their work, Bashmakova and Smirnova write:

Modern history of mathematics seems to be dominated by the view that up to the 1830 s the mainspring of the development of algebra was the investigation and solution of determinate algebraic equations, and especially their solution by radicals. We will show that this viewpoint is one-sided and gives a distorted representation of its evolution. In short, we claim that the role of indeterminate equations in the development of algebra was no less important than that of determinate equations. (Bashmakova and Smirnova 2000, p. xv.)

23 van der Waerden's text was still being referred to in algebra courses, even when I was a graduate student in mathematics. Also, a referee informed me that van der Waerden's book was used as the textbook for an abstract algebra course he attended in the nineties. 
mathematics". ${ }^{24}$ One can proceed by first giving the definitional axioms of a group as follows:

A group is a system consisting of set and an operation on the set such that: (a) the set is closed under this operation; (b) the operation is associative; (c) the set has a right identity element; and (d) every element of the set has a right inverse.

An example of a group usually given is the integers under addition. Then, after proving from the axioms the basic features of all groups, the term 'ring' can be defined as follows:

A ring is a system consisting of a set $S$ and two binary operations on $S$, plus (symbolized ' + ') and times (symbolized ' $x$ '), such that:

(1) the system consisting of $S$ with the operation plus is a commutative group;

(2) $S$ is closed under the operation times, and times is associative;

(3) for every $a, b$, and $c$ in $S$,

$$
\begin{aligned}
& a \times(b+c)=(a \times b)+(a+c) \\
& (b+c) \times a=(b \times a)+(c \times a)
\end{aligned}
$$

An example of a ring that is frequently given is the integers under addition and multiplication. One can then apply to that part of a ring consisting of $S$ with plus all that was learned earlier about abstract groups. For example, it can be immediately inferred that, in a ring, there is only one additive identity; that any element's right inverse is unique and is identical to the element's left inverse; and that the inverse-of-a-sum law holds - that is, the inverse of a sum of elements is identical to sum of the inverses of the elements, taken in reverse order. Clearly, much redundancy of exposition and learning can, in this way, be eliminated. The effectiveness of this sort of efficiency is illustrated by the following example.

\section{Nonstandard analysis}

Nonstandard analysis was developed by Abraham Robinson in the 1960s to provide a "framework for the development of the Differential and Integral Calculus by means of infinitely small and infinitely large numbers" 25 (Robinson 1996, p. xiii). Infinitely small numbers (or infinitesimals), as well as infinitely large numbers, can be introduced by first defining the real numbers as equivalence classes of Cauchy sequences

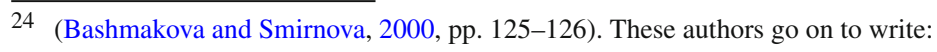

Group theory plays a crucial role in quantum physics. Wolfang Pauli, one of the creators of quantum physics, wrote that the ideas of group theory belong to "the most powerful instruments of modern physics", and that, in his view, their fruitfulness is "far from having been exhausted" (W. Pauli, Collected Scientific Papers, ed. R. Kronic and W. F. Weisskopf, 2 vols., Interscience, 1964). The subsequent evolution of physics brought with it a splendid confirmation of Pauli's words. (pp. 126-127)

25 This framework was used to develop more advanced branches of analysis, such as Functional Analysis and the theory of functions of complex variables. 
of rational numbers and then introducing an equivalence relation over arbitrary sequences (not necessarily Cauchy) of real numbers to specify a new totality of numbers (the "hyperreal numbers") which are defined to be certain equivalence classes of these sequences of real numbers. ${ }^{26}$ An ordering relation can then be defined over the hyperreals, and then proved to be a linear ordering. Addition and multiplication of the hyperreals can be so defined as to satisfy the usual algebraic laws. ${ }^{27}$

Among these hyperreal numbers, there are numbers that, by the above mentioned linear ordering, are positive (greater than zero) but less than every positive real number. Such numbers are called 'positive infinitesimals'. In addition to the positive infinitesimals, there are negative infinitesimals. A hyperreal number is an infinitesimal if it is a positive infinitesimal, zero, or a negative infinitesimal.

To see how the introduction of infinitely small numbers can mesh with clear intuitions about fundamental concepts in the Differential and Integral Calculus, Robinson gave the following example:

[J] ust as the (mean) velocity moving along a straight line between times $t_{1}$ and $t_{2}$ is given by

$$
\left(s_{2}-s_{1}\right) /\left(t_{2}-t_{1}\right)
$$

where $s_{2}$ and $s_{1}$ are the corresponding displacements of the particle relative to a fixed point, so one is inclined intuitively to define the instantaneous velocity of the particle at time $t_{1}$ by the same ratio, where $t_{2}-t_{1}$ and hence $s_{2}-s_{1}$ are now supposed to be infinitely small. ${ }^{28}$

Noting that Leibniz adopted just such an approach to his calculus, Robinson concluded with the comment that "a method which had been given up as untenable has at last turned out to be workable" (Robinson 1973, p. 16).

Now the algebraic structure described above can be proved to be a non-Archimedean ordered field. ${ }^{29}$ One can then apply to the structure of the hyperreal numbers everything proved in abstract algebra about fields, thus shortening the needed proofs and theoretical exposition of this novel structure. In particular, since a field is a special kind of ring, ${ }^{30}$ one can infer immediately that the inverse-of-a-sum law holds for addition of the hyperreal numbers.

Essentially everything I said above about algebraic structures is acceptable to classical mathematicians - but not to the fictionalist. The ring of integers under addition and multiplication is a set of numbers, and hence is, for Field and his followers, a mere fictions that has no more reality than unicorns and Chimeras, and the mathematical theorems about them are taken to be like the sentences in a work of fiction: not appropriately evaluated for truth or falsehood. What, then, could be the rationale for developing an algebraic theory consisting of a great many non-factual sentences about fictional objects that are not appropriately called true or false? One cannot easily

\footnotetext{
26 The equivalence relation is called by H. J. Keisler "an ultraproduct equivalence relation", asserting that such a relation can be proved to exists (Keisler 1976, p. 879).

27 Sums and products are defined in the usual way of defining operations with Cauchy sequences.

28 (Robinson, 1973, p. 16).

29 See (Davis, 2005, pp. 45-56), and (Keisler, 1971, p. 822).

30 A ring is a field if it satisfies the following conditions: (1) the operation $\times$ (multiplication) is commutative; (2) it has at least two elements; (3) it contains a multiplicative identity; (4) it has no proper divisors of zero (i.e. the additive identity); (5) every element that differs from zero has a multiplicative inverse.
} 
argue that the structural view has proved to be fruitful in mathematics because that sort of algebra turns out to be conservative over nominalistic theories. Since the structural approach to algebra was justified by its fruitfulness in pure mathematics, where the distinction between nominalistic and platonic languages needed in the proof of the Conservation Principle is simply not made, such a strategy does not adequately account for the universal acceptance of the modern algebraic approach by pure mathematicians, most of whom were not especially concerned with applying algebra to science. ${ }^{31}$

\section{Groups of transformations}

Let $S$ be a set and consider the set of 1-1 transformations of $S$ onto itself. This set of transformations of $S$ forms the basis for a well-known group. We can define an operation (to be called 'addition') involving these transformations as follows: Let $O$ be any ordering of $S$. If $P_{1}$ and $P_{2}$ are such transformations, then let $P_{1}+P_{2}$ be the ordering that results from first applying transformation $P_{1}$ on $O$ and then applying transformations $P_{2}$ on the ordering that results from the first transformation. It can be seen that $P_{1}+P_{2}$ will result in an ordering identical to what results from performing some one of the possible $n$ ! transformations, say $P_{k}$, of the original ordering $O$. Thus, we can take $P_{1}+P_{2}$ to be identical to $P_{k}$. In short, the collection of these transformations can be regarded as closed under this sort of addition. It is a trivial matter to go on to show that this operation of addition is associative. Also, the trivial transformation that leaves the position of everything unchanged can be specified to be the identity transformations (or the identity element of the addition operation). Since for every transformation $P$, there is a transformation that undoes the changes that $P$ makes, one can conclude that every transformation has a right inverse, and hence that this system is a group. ${ }^{32}$

We can immediately infer that all the usual properties of groups hold of this system. We can infer, for example, that the identity element is unique; that any element's right inverse is also unique and is identical to the element's left inverse. We can also conclude that the inverse of $\left(P_{1}+P_{2}\right)$ is identical to (the inverse of $P_{2}+$ the inverse of $\left.P_{1}\right)$.

Groups of transformations are particularly important in algebra because of the following fundamental theorem (due to Arthur Cayley):

Every group is isomorphic to some transformation group. ${ }^{33}$

\footnotetext{
31 This is not to suggest that the fictionalist cannot come up with some sort of explanation of the development of modern algebra in terms of conservatism. However, any such explanation, I believe, will be neither adequate nor very plausible.

32 Lest one think that this example is esoteric and artificial, it should be noted that transformation groups play an important role in geometry - furthermore, the study of transformation groups "provided the original impetus to the development of the theory of groups" (Jacobson 1951, p. 15).

33 See (Jacobson, 1951, pp. 28-29), for a proof of this theorem.
} 


\section{A quasi-model of group theory}

Imagine now that eight wooden blocks have been lined up to produce a linear ordering. Each of the 8 ! (or 40,320) transformations of the set of blocks can be regarded as yielding a permutation of the original ordering. Thus, if $t(x)$ is such a 1-1 transformation, then just think of replacing each $x$ in the original ordering with $t(x)$ : the ordering of the set that results from these replacements yields a reordering that is a permutation of the original ordering — so long as there is some block $y$ which is not the same as $t(y)$. For the case of the identity transformation, we do not obtain a true reordering, but for sake of simplicity of discussion, we regard the identity transformation as yielding a permutation. Thus, we will say that there are 8 ! (or 40,320) permutations of this ordering.

We shall define addition of the these permutations as we did earlier for the permutation groups, except now we say that $P_{1}+P_{2}$ is the ordering that results when first the permutation $P_{1}$ is actually performed by some competent agent armed with a suitably programmed computer ${ }^{34}$ and then $P_{2}$ is actually performed by that same agent on the ordering that results from the first permutation. We now have a quasi-model of group theory.

Why do I call it a "quasi-model" (and not a model)? Well, defining the addition operation in terms of what results from an actual agent performing various permutations makes the operation dependent upon the powers of the agent and the computer used. If the agent were god-like, then perhaps we would have a model of group theory, but since we are concerned here with an actual (human) agent, we need to take account of the possibility that the agent may commit errors or become completely exhausted at some time. Thus, we are not in a position to assert that all the axioms of groups theory are true under the interpretation, and we do not have a genuine model but only a quasi-model. The situation is not unlike the case of an actual computer modeling a Turing machine: since any actual computer is liable to make errors or break down and are limited in the number of operations it can perform, they cannot be true Turing machines, but they can mimic a Turing machine in various ways. Similarly, the above quasi-model can be used to model permutation groups in certain ways.

Consider now the theorem of group theory: "Every element has only one right inverse". Using the above quasi-model, we can test the truth of the theorem by examining any number of specific cases of right inverses of permutation sums and seeing if what the law states does indeed hold. (Actually, most mathematicians will be able to see from a single case that the theorem will hold for every case). So does not this result suggest that the theorem of group theory has a content that can be tested and verified?

This time, consider the inverse-of-a-sum law of groups. We could take any two permutations $P_{1}$ and $P_{2}$ of the ordered eight wooden blocks and then perform the sum of permutations $P_{1}+P_{2}$. We could then determine the inverse permutation, say $P_{j}$, of the sum. We could also determine which permutation, say $P_{k}$, is the sum of the inverse of

\footnotetext{
${ }^{34}$ It can be stipulated that the computer is programmed to: (a) output the inverse of a permutation $x$ of the blocks, when $x$ is inputted; and (b) output the sum of $x$ and $y$, when the two permutations $x$ and $y$ are inputted.
} 
$P_{2}+$ the inverse of $P_{1}$. As in the previous case, finding that $P_{j}=P_{k}$ would constitute a verification of the truth of the inverse-of-a-sum law. Of course, we could verify the truth of the theorem additionally by taking another pair of permutations and repeating the above verification for that pair. Indeed, we could, in theory, test the theorem for all possible pairs of these permutations and thus get a very convincing verification of the inverse-of-a-sum law. Clearly, the inverse of a sum law can be repeatedly tested and found to be true, very much like a statement of an empirical science. Of course, verifications of this sort have in fact been carried out by countless students throughout the world, using essentially the above type of quasi-model. Such verifications cast considerable doubt on Field's thesis [NT] that no part of mathematics is true. ${ }^{35}$ Since Field has produced no good reason to deny that the theorem is true, the preponderance of evidence is surely on the side of the mathematicians who believe that the theorem is true. ${ }^{36}$ We could devise similar verifications of many of the theorems of group theory to undermine the plausibility of Field's [NT].

What about Field's deflationist thesis [MK]? Field's only reason for espousing the view that, apart from some empirical knowledge of what mathematicians may assert, what distinguishes the mathematician who knows a lot of mathematics from someone who knows little or no mathematics is modal knowledge of the two forms listed earlier. But does the algebraist's knowledge of the inverse of a sum law of group theory consist merely in the knowledge of what other mathematicians may assert? Let (*) be statement:

In group theory, the inverse of a sum is equal to the sum of the inverses of the summands taken in reverse order.

Surely it would be a mischaracterization to describe the algebraist's knowledge of the truth of $(*)$ as knowledge of what other mathematicians will respond to various questions about inverses. For, even if she believed that all other mathematicians had somehow had their knowledge of the inverse of the sum law completely erased by an evil demon, she could still know that (*) is true. Then should we maintain that the algebraist's knowledge of the truth of (*) must be knowledge of logical necessity of one of the two types Field listed? Most specifically, must the mathematician's knowledge be knowledge that it is logically necessary that, in group theory, the inverse of a sum is equal to the sum of the inverses of the summands taken in reverse order? Here, I must recall to your attention that, as Field articulates his concept of logical necessity, (*) is not logically necessary. (Recall that, as Field defines 'logical necessity', it is not logically necessary that all bachelors are unmarried).

Besides, even if $(*)$ were logically necessary, still (*) expresses a proposition distinct from:

\footnotetext{
35 Those who have studied my book (Chihara 2004) may wonder how I can allow that the theorem about inverses of sums is true. Such readers should note that the theorem expresses a feature of the group structures that is essentially identical to what the sentence expressing the structural content of the theorem does.

36 Before concluding, from a rejection of the Indispensability Argument, that there is no reason to believe that any part of mathematics is true, one should surely investigate why a great many, if not practically all, mathematicians believe that much of mathematics is, in some way, true. Such a common and widespread belief cannot be based upon the Indispensability Argument.
} 
(**) It is logically necessary that in group theory, the inverse of a sum is equal

to the sum of the inverses of the summands taken in reverse order.

(*) and (**) express different propositions, and some mathematicians could know (*) without knowing $(* *)$. Thus, we can see that difference between the set of propositions known by mathematicians and the set of propositions known by non-mathematicians will include more than propositions of the two types Fields specifies.

The above discussion shows the dubiousness of Field's two theses [NT] and [MK]. It also underlines the importance of the specific content possessed by an individual theorem-something that tends to be overlooked by those accepting the theoretical juice extractor view. ${ }^{37}$ After all, what the algebraist infers from the inverse- of-sum law is quite different from what she infers from the uniqueness-of-the-right-inverse theorem, and she would verify the former theorem in a way that is very different from the way she would verify the latter theorem. Do we not, then, have compelling reasons to think that the inverse-of-a-sum theorem has a specific content which tells us something quite definite about inverses of sums of permutations of arbitrary sequences of these blocks? The omission, in Field's account, of this striking feature of individual theorems is undoubtedly due to the fact that his fictionalist understanding of mathematics was developed almost solely in response to the indispensability argument-a response which required no role at all to be played by the contents of individual theorems. We can see now how a philosopher of mathematics, by focusing so intently and single-mindedly on just one problem or contentious issue in the philosophy of mathematics, can be led to assert and to maintain a doctrine as implausible as Field's that "there is no reason to regard any part of mathematics as true".

\section{How Field might attempt to revise his account}

David Etlin suggested at my talk that Field might respond to my objections by revising his position to allow some theorems of mathematics to be true: according to the revised position, only those theorems that explicitly assert the existence of mathematical objects would be counted as false. Thus, the theorem asserting that every group has a unique identity could be classified as true, since it does not explicitly assert the existence of any mathematical objects.

Now let us look more carefully at the suggested revision. The idea is to classify as false only those theorems that explicitly assert the existence of mathematical objects. However, it can be easily established that this revised fictionalism will be saddled with serious problems arising from the fact that sets of mathematical theorems, all the members of which would be classified as true, could still imply the existence of mathematical objects and thereby entail what would be classified as a false statement. For example, the following theorems of set theory would be classified as true, since neither explicitly asserts the existence of any mathematical objects:

If no unit set is empty, then there is an empty set.

37 Cf. the idea of the structural content of a mathematical theorem discussed in great detail in my (Chihara
2004). 
No unit set is empty.

But the conjunction of the two implies that there is an empty set, which must be classified as not true. This shows that the revised-fictionalist must come up with a better way of distinguishing the theorems that are to be classified as true from those that are to be classified as false, if she is to avoid the sort of problems I have indicated above.

A reviewer for this journal has suggested the following way of revising Field's account of mathematics:

\begin{abstract}
The obvious modification is to claim that some non-existence entailing mathematical claims are true .... [T] he most natural suggestion is to treat mathematical predicates as having empty extensions and mathematical names as empty names. On this view, some non-theorems of (for instance) Peano Arithmetic are true. For instance, the claim "Every number is prime" is true, since there are no numbers .... There is, of course, a serious question about how to handle empty names in logic, but this does not pose a special problem for the case of mathematics, since it arises in non-mathematical contexts, too. Whatever free logic we adopt for non-mathematical contexts can be extended to mathematics.
\end{abstract}

I would now like to respond to this suggestion first by making my position clear. I certainly never claimed, nor did I ever suggest, that there is no way for Field to revise his account of mathematics so as to obviate my objections above, while preserving his basic position on the conservatism of classical mathematics. No doubt, there are many different ways of devising such a revision. But one of the main points of my paper was to show that developing one's view of mathematics by focusing almost solely on applications of mathematic in science can lead to a distorted understanding of the nature of mathematics. Also, I wished to show that, had Field explored more thoroughly features of pure mathematics, he probably would have adopted a significantly different brand of nominalism. These points, I shall argue, are not undermined by any such revision.

If Field were to accept the reviewer's suggestion, he could not continue to hold that his account of mathematics does not require any "reinterpretation" of mathematics. Interpreting mathematics in the way being suggested certainly does require a significant reinterpretation of mathematics. It is clear that practicing mathematician do not understand mathematical sentences in the way being suggested.

Consider the reviewer's suggestion regarding the form of free logic to be employed in mathematical contexts. Evidently, following the suggested revision would involve changes in our practices that would make the mathematician's theorizing even more complicated and more difficult than it is. Certainly, these are not changes in our mathematical practices that one should advocated lightly.

Field's account of mathematics, if revised in the way suggested, would not be in perfect agreement with our present mathematical practices. Contemporary mathematicians do not assert that such sentences as 'All natural numbers are prime' and 'Every set has a member' are true. Nor do they maintain that 'There are infinitely many prime numbers' is a false statement. The revised position simply would not fit the actual practices of contemporary mathematicians.

Also, these revisions would require Field to abandon his "fictionalism", according to which it is not appropriate to assess mathematical theories in terms of truth and falsehood: since, under the suggested revision, many, if not all, the statements of 
mathematics are either true or false, it would indeed be appropriate to evaluate such theories in terms of truth and falsehood. ${ }^{38}$

The abandonment of his fictionalism would also engender trouble for Field's deflationist account of mathematical knowledge. This is because, according to the suggested revision, there would be many mathematical truths - truths expressed by, for example, theorems of group theory - that are known to be true by mathematicians but not known to be true by non-mathematicians. For example, $(*)$ would express a truth, but $(* *)$ would not; and, as was shown earlier, (*) would not be of either of the two modal forms specified by Field's deflationist account. In short, accepting the reviewer's suggested revision would require the abandonment, or significant revision, of Field's deflationist account.

\section{A reexamination of the claim that fictionalism is superior to all its rivals}

I would like now to return to Balaguer's argument for the superiority of fictionalism over all its nominalistic rivals. Recall that this reasoning was based, in part, on his assertion that there is nothing in mathematical practice that runs counter to fictionalism. To test this assertion, I should now like to reconsider the example of nonstandard analysis.

One of the central principles of nonstandard analysis is given by the Transfer Principle - a theorem, really, that tells us very roughly that there is an extension of the real numbers that includes the infinitesimals of nonstandard analysis and that has the same properties as the standard real numbers in so far as those properties can be expressed in a certain formal language. ${ }^{39}$ To give an account that is a bit less rough, and focusing on a simple form of the principle, ${ }^{40}$ let us suppose that a formal quantificational language $L$ is specified for referring to the sets of Zermelo-Fraenkel set theory-a language for which the semantics is recursively given in a standard way, such that, for any (closed) sentence $s$ of the language, and any structure $M$ of the appropriate sort, $s$ must be either true in $M$ or false in $M$. Then, if $s$ is a sentence of $L$ in which the only constants are individual constants (i.e. constants that denote only "individuals"-i.e. urelements or non-sets of the structures $M_{1}$ and $M_{2}$ ), and if $M_{1}$ is a structure appropriate for $L$ with a universe whose real numbers are the standard ones, and $M_{2}$ is a structure appropriate for $L$ with a universe whose "real" numbers are the nonstandard hyperreal numbers, then

$s$ is true in $M_{1}$ iff $s$ is true in $M_{2}$

Davis points out that the Transfer Principle "is typically used by first proving a desired result in the nonstandard universe, and then, noting that the result is express-

\footnotetext{
38 It should be noted that the revised version would also not fit Balaguer's notion of " fictionalism", according to which the sentences of mathematics (and hence the theorems of mathematics) are like the sentences of fictional works in which references are made to non-existent objects and so are regarded as not true (Balaguer 1998, p. 12). Thus, Balaguer's fictionalist would classify all theorems containing reference to groups as not true.

39 See (Davis, 2005, p. 2).

40 For a precise statement of the theorem, see (Davis, 2005, pp. 22-28).
} 
ible in the language [ $L$ in the above discussion of the Transfer Principle], concluding that it holds in the standard universe as well" (Davis 2005, p. 3). Now is this mathematical practice compatible with the fictionalist's view? Well, for the fictionalist, the Transfer Principle is not true. So the fictionalist cannot appeal to the theorem to justify drawing the type of inference Davis described above, and it would seem that the fictionalist is in no position to accept the reasonableness of the above mathematical practice. Evidently, contrary to what Balaguer has claimed, there are some mathematical practices that do run counter to the fictionalist's position.

Both Greg Ray (at the conference) and a reviewer have suggested a response to the above objection. Cannot the Transfer Principle be proved within ZFU? If so, couldn't Field argue, by appealing to the conservatism of ZFU, that he would be justified in using the principle to draw nominalistic conclusions from nominalistic theories? Well, strictly speaking, the Transfer Principle cannot be proved within ZFU alone, since ZFU can only refer to sets and not to all the things that the principle talks about. Of course, one could prove in a suitable metatheory that certain number-theoretic relations, such as the proof relation, can be represented in ZFU. Then, using the device of Gödel numbering, one can, in a sense, "express" such statements as "Sentence $S$ is provable in ZFU". So there might be some hope that, by using such metamathematical devices, even the Transfer Principle could be expressed and proved in ZFU.

Let (A) be the statement:

The Transfer Principle is provable in ZFU.

Now Field holds that no part of mathematics is true. So even if (A) were proved in some metamathematical theory, Field would not be able to conclude that (A) is true. Besides, Field would not be in a position to know that all the recursively decidable number-theoretic relations and predicates that are needed to express the Transfer Principle in ZFU are indeed representable in ZFU. This is because he would not be in a position to assert the truth of the theorems of the metamathematical system that are needed to prove the Transfer Principle in ZFU in the way being envisaged. Specifically, various number-theoretic theorems, such as the Chinese Remainder theorem, that are used in proving the representation theorem cannot be claimed by Field to be true. Thus, it is hard to see how he could claim to prove (A).

Perhaps it will be maintained that Field does not have to know that (A) is true or even that the Transfer Principle is true. He needs only to construct a first-order derivation from the axioms of ZFU of the "Transfer Principle" (that is, the set theoretical statement that, supposedly, can be shown to express via coding the Transfer Principle), and then he could use the Conservatism of ZFU to justify using the Transfer Principle to derive nominalistic conclusions from nominalistic theories.

Well, this strategy won't work for a variety of reasons. First of all, nobody is going to construct such a first-order derivation of the "Transfer Principle": it is simply not practically possible. Besides, even if, by some miracle, some one did actually construct such a derivation, no one could realize that the sentence derived did express the Transfer Principle, without relying on many theorems of proof theory-in effect relying on the truth of those theorems. Furthermore, it is not obvious such a derivation is even possible. Even to construct a sentence of ZFU that expresses the Transfer Principle would require some rather powerful concepts, such as the relations $x$ is true in 
structure $y$ and structure $z$ satisfies the sentence $w$-relations that are not effectively decidable and hence not representable in Peano Arithmetic.

Another strategy would be to attempt to formulate a metamathematical theory, say $M$, that includes ZFU as a subtheory, and that has a vocabulary (with the appropriate rules of inference involving its terms) which is sufficiently strong to permit one both to express the Transfer Principle directly (without having to use proof theoretical devices) and also to derive the Principle from the axioms of $M$. In short, one could attempt to formalize, in first-order logic, the usual model theoretic proof of the Transfer Principle. Then, if one could prove that $M$ is conservative over nominalistic theories, the applied mathematician could use this Transfer Principle to derive in $M$ nominalistic conclusions from nominalistic theories formalizable in $\mathrm{M}$, without having to maintain that the Principle is true. ${ }^{41}$

A serious problem with this suggestion is the hurdle of proving the conservatism of $M$. No one has proved such a Conservation Principle for any theory as complicated as $M$ would have to be. And given the powerful model-theoretic semantical notions that would have to be formalized in $M$, it is not clear that such a theorem could be proved. Certainly, the kind of proof Field gave of the conservation of ZFU over nominalistic theories could not be simply carried over for this case. Field would have to deal with a much more complicated theory using $M$ as the target theory than he did using ZFU.

But even if one could prove the conservatism of $M$, another problem would have to be faced. Supposing that the Transfer Principle were provable in M, the Conservation Principle, however, would not: it would be a sentence of a metatheory of M. Any applied mathematician or scientist willing to use the Transfer Principle in the way being suggested would have to have some assurance that the Principle could be reliably used in that way. But could the fictionalist offer such an assurance based on his proof of the Conservation Principle? How could he? First of all, the axioms of any metatheory used to prove conservation would undoubtedly be nominalistically unacceptable. Besides, according to Field, the very statement of the Conservation Principle is not supposed to be factual. For the fictionalist, the statement of the principle is like a sentence in a work of fiction: neither true nor false.

More generally, one can see that Fictionalism runs into problems whenever model theory is employed to justify using a metalogical theorem to draw inferences. Not surprisingly, Field has been forced by this problem to resort to some unusual logical maneuvers. Consider, for example, his use of model theory to prove the conservatism of ZFU. How was he able to square such a proof with his fictionalistic view of mathematical theorems? He claimed that the model theory was used only as an assumption in a kind of reductio ad absurdum argument against his platonic opponents. Thus, he wrote: "if I am successful in proving platonistically that abstract entities are not needed for ordinary inferences about the physical world or for science, then anyone who wants to argue for Platonism will be unable to rely on the Quinean argument that the existence of abstract entities is an indispensable assumption" (Field 1980, p. 6). It

\footnotetext{
41 This is basically the strategy suggested by a referee, who wrote: "I don't see why Field cannot just make use of standard mathematics and metamathematics, all understood to be conservative over nominalistic theories." The problem, of course, is the idea that Field can just take all that metamathematics to be conservative over nominalistic theories.
} 
is clear that Field thus saw himself as not relying upon his model theory to arrive at the knowledge of a mathematical truth. ${ }^{42}$

But did he not make use of such metalogical theorems as the completeness and soundness theorems of first-order logic in his theorizing about the nature of space? For example, he wrote:

$[R]$ ecall that conservativeness as I defined it initially is a semantic notion, ... but in referring to some of the arguments as proof-theoretic, and in the way I wrote the proof in note 15, I showed that it was the syntactic notion I was dealing with. The justification for the shift from semantic to syntactic notions is of course the Gödel completeness theorem for first-order logic. (Field 1980, p. 115, n. 30)

The above reasoning illustrates how Field frequently justified drawing conclusions by appealing to metalogical theorems - conclusions that could only be legitimately drawn if the truth of the theorem cited were justified. In other words, he Frequently treated metalogical theorems, not as mere sentences in a work of fiction, but rather as expressing proved propositions. But to do so simply does not fit his avowed fictionalist stance. It was, perhaps, in an attempt to justify at least some such cases of appealing to metalogical theorems that Field developed a kind of ersatz semantic theory. Thus, he published an article attempting to show that a nominalist can legitimately use certain metalogical theorems of model theory, such as the completeness and soundness theorems of first-order logic (Field 1989). Field's basic strategy in developing his ersatz semantic theory is to utilize certain modal principles which, he argues, enable him to justify his use of his modal versions of the completeness and soundness theorems.

That Field's ersatz justification of his use of metalogical theorems is cogent and reasonable can, I believe, be doubted, ${ }^{43}$ but in any case, it easy to see that there is nothing in it to justify an acceptance of the kind of complex set theoretical models required to generate nonstandard analysis, let alone the high-powered models used in much of contemporary model theory. ${ }^{44}$ Furthermore, the fact that Field has to resort to ersatz versions of soundness and completeness in the above way exposes the implausibility of Balaguer's claim that there are no mathematical practices that run counter to the fictionalist's view of mathematics. For we have seen that whenever standard model theory is used in classical mathematics to draw some conclusions about what is provable or what must hold in some structure, Field is forced to rely upon his own version of semantic theory to try to capture what was concluded by the classical

\footnotetext{
42 For more on this topic, see (Chihara, 1990, pp. 320-321).

43 See my (Chihara 2004, Chap. 11), especially Sect. 3, for my doubts about his justification.

44 This defect in Field's account of mathematics is striking similar to a defect in Graeme Forbes's antirealist account of modal logic. An odd feature of Forbes's account is the absence of any sort of significant role for the mathematical structures of standard possible worlds semantics to play. See, for example, my (Chihara 1998), where I wrote:
}

[A]lmost all of Forbes's account is focused on the task of explaining how possible worlds sentences can be used to assess the validity and invalidity of modal arguments. But possible worlds semantics is used for much more than assessing validity and invalidity, just as the model theory of first-order logic is concerned with much more than just the evaluation of the validity of arguments. In the case of first-order logic, for example, the Homomorphism Theorem is applied to provide information about definability within structures, elementarily equivalent structures, and decidability. (p. 167) 
mathematician, thus relying upon just the sort of "controversial, non-standard, nonface-value interpretations that seem to fly in the face of actual mathematical practice" that Balaguer attributes to the rivals of fictionalism.

Well, might not Field try to employ the reductio technique that he used in (Field 1980) to answer my present objections to Balaguer's thesis? More specifically, might he not attempt to use the strategy of reductio ad absurdum to justify his acceptance of the mathematical practice described by Davis? How would that strategy work? Perhaps we can start by assuming, as part of the reductio strategy, that the platonic model theory $\mathbf{M}$ is true. Then, from this assumption, one can prove in $\mathbf{M}$ the truth of the Transfer Principle. One can then infer, based upon the assumption, the soundness of the practice described by Davis. But what follows from that? What is the absurdity? How do we arrive at what is wanted?

Perhaps we should attempt a more direct reductio argument for the validity of the mathematical practice described by Davis. In other words, assume that that mathematical practice is not valid, and try to deduce a logical absurdity of some sort. Where does that get us? Nowhere so far as I can see.

The lack of a genuine model theory in Field's account of mathematics adequate for even nonstandard analysis points to a serious defect, since (as I indicated earlier) the heavy appeal to structures and models is one of the most common and important features of contemporary mathematics. ${ }^{45}$ My own structural account of mathematics was fashioned to provide the nominalist with the material to understand such uses of structure in mathematics. ${ }^{46}$

\section{Conclusion}

My aim in this paper was to show how developing one's view of mathematics by focusing almost solely on applications of mathematic in science can lead to a very distorted understanding of the nature of mathematics. This is because, although applications of mathematics can teach us much about the nature of mathematics, there is also much to be learned about mathematics from considerations of pure mathematics, where matters of applications carry no weight at all. ${ }^{47}$ There clearly needs to be, by

\footnotetext{
45 An indication of the importance of structure for contemporary mathematics is to be found in the enormous body of work published under the name 'Nicolas Bourbaki' - "the collective pseudonym of a changing and secret group of mathematicians, most of them French, who have collaborated since the 1930s with the intention of achieving a complete and definitive compilation of mathematical knowledge" (Borowski and Borwein 1991, p. 60). This group has emphasized the importance of structure in mathematics, publishing more than 36 volumes of mathematics, in which they classify the various areas of mathematics in terms of structure.

46 See Chihara (2004), especially Chaps. 7 and 8.

47 Hardy was an illustrious mathematician who worked almost exclusively in pure mathematic: primarily analysis and number theory (see Newman's commentary on Hardy in (Newman 1956, p. 2024)). He assessed his own mathematical contributions to human knowledge with the words:
}

I have never done anything 'useful.' No discovery of mine has made, or is likely to make, directly or indirectly, for good or ill, the least difference to the amenity of the world. (Newman 1956, p. 2026) 
philosophers of mathematics, a careful investigation of pure mathematics, if anything like an adequate account of mathematics is to be produced.

Open Access This article is distributed under the terms of the Creative Commons Attribution Noncommercial License which permits any noncommercial use, distribution, and reproduction in any medium, provided the original author(s) and source are credited.

\section{References}

Balaguer, M. (1998). Platonism and anti-platonism in mathematics. Oxford: Oxford University Press.

Bashmakova, I., \& Smirnova, G. (2000). The beginnings and evolution of algebra. The Mathematical Association of America.

Borowski, E. J., \& Borwein, J. M. (1991). The Harper Collins dictionary of mathematics. New York: Harper Collins Publishers.

Chihara, C. S. (1973). Ontology and the vicious-circle principle. Ithaca: Cornell University Press.

Chihara, C. S. (1989). Tharp's 'Myth and Mathematics'. Synthese, 81, 153-165.

Chihara, C. S. (1990). Constructibility and mathematical existence. Oxford: Oxford University Press.

Chihara, C. S. (1998). The worlds of possibility: Modal realism and the semantics of modal logic. Oxford: Oxford University Press.

Chihara, C. S. (2004). A structural account of mathematics. Oxford: Oxford University Press.

Corry, L. (1996). Modern algebra and the rise of mathematical structures. Basel, Boston, Berlin: Birkhauser Verlag.

Davis, M. (2005). Applied nonstandard analysis. Mineola, NY: Dover Publications.

Field, H. (1980). Science without numbers. Princeton: Princeton University Press.

Field, H. (1984). Is mathematical knowledge just logical knowledge? Philosophical Review, 93, 509-552.

Field, H. (1989). Is mathematical knowledge just logical knowledge? In Realism, mathematics and modality (pp. 79-124). Oxford: Oxford University Press.

Field, H. (1992). A nominalistic proof of the conservativeness of set theory. Journal of Philosophical Logic, $21,11-23$.

Hardy, G. H. (1941). A mathematician's apology. Cambridge, England: Cambridge University Press.

Jacobson, N. (1951). Lectures in abstract algebra. New York: D. Van Nostrand Company.

Keisler, H. J. (1971). Elementary calculus: An approach using infinitesimals (Experimental Version). Tarrytown-on Hudson, N.Y.: Bodgen and Quigley.

Keisler, H. J. (1976). Elementary calculus. Boston: Prindle, Weber \& Schmidt.

Newman, J. R. (Ed.) (1956). The world of mathematics. New York: Simon and Schuster.

Robinson, A. (1973). Numbers - What are they and what are they good for? Yale Scientific, 47, 14-16.

Robinson, A. (1996). Non-standard analysis. Princeton: Princeton University Press.

Tharp, L. (1989). Myth and mathematics: A conceptualistic philosophy of mathematics. Synthese, 81, 139-201.

Footnote 47 continued

Interestingly, in 1908, he wrote a letter to Science on a problem involving the transmission of dominant and recessive Mendelian characters in a mixed population. In the letter, he showed that the proportion of dominant and recessive genes in a randomly mating population remains constant unless there are outside influences. As this law of population genetics was independently discovered by Wilhelm Weinberg, it became known as the Hardy- Weinberg law. Ironically, this law has become one of the central principles of population genetics! 